\title{
Evaluating the shear resistance and ultimate dewatering performance of polymer-treated tailings
}

\author{
L Boxill BASF Canada Inc., Canada \\ A Costine CSIRO Mineral Resources, Australia \\ P Fawell CSIRO Mineral Resources, Australia \\ M Catling BASF Corp., USA
}

J Bellwood BASF plc, UK

\begin{abstract}
Anionic copolymers have been used to enhance the dewatering and strength development properties of a wide range of tailings substrates around the mining world for at least two decades. The evaluation and selection of polymer treatment candidates for field application is often based on results obtained from a series of screening experiments to satisfy specified key performance indicators (KPIs). However, if the screening protocol or stated KPIs fail to account for the shear environment the treated tailings will experience during polymer dosing and prior to deposition, what often results is the incorrect selection of the polymer to achieve the desired dewatering and performance criteria post-deposition.

Results from several laboratory-scale screening experiments are used to demonstrate how tailings (both analogues and industrial samples), dosed with the same polymer(s), can exhibit widely different characteristics when subjected to distinct shear conditions. This paper will also show how polymer selection would change depending on whether results from conventional screening criteria were used versus results obtained from an expanded screening protocol that accounts for some of the shear characteristics of the tailings treatment and deposition environment.
\end{abstract}

Keywords: tailings polymer treatment, topological mixing, controlled shear, conditioning time

\section{Introduction}

Evaluation of the effects of shear on polymer-treated slurry systems is an important consideration, as the fabric and structure of the resulting aggregates is a major factor in the rate of dewatering, strength development, and overall geotechnical performance of the treated substrate (Boxill 2016; Beier et al. 2013; McFarlane et al. 2006). Fundamentally, the shear response of a polymer is affected by its molecular weight which impacts the viscosity exhibited by the polymer in solution at a given concentration. Increasingly, accounting for the shear condition of tailings substrates prior to treatment and the shear environments during flocculation, transportation and deposition is being identified as critical to achieving the desired characteristics of deposited treated materials. Unfortunately, key performance indicators (KPIs) that only account for shear conditions at a single stage of a process often fail to account for shear effects further downstream. This often results in incorrect selection of treatment chemistry, producing suboptimal or undesirable characteristics of polymer-treated substrates post-deposition. As a consequence, clients are then frustrated when desired post-depositional tailings characteristics and performance are not satisfied.

This paper explores shear effects produced when polymers of similar anionic charge, but varying molecular weight are used to treat model tailings substrates. The low solids content slurry was a tailings analogue (6 wt\%) comprised of a mixture of kaolinite, silica and sand particles. The high solids content slurry was $21 \mathrm{wt} \%$ with a similar mineralogical composition. To allow both slurries to mimic the characteristics of typical tailings process fluids, the low and high-solids slurries were made up in $0.035 \mathrm{M} \mathrm{NaCl}$ and $0.005 \mathrm{M} \mathrm{CaCl}_{2}$, 
respectively. Findings from some preliminary work investigating the effects of shear on two polymers of similar molecular weight during flocculation of two oil sands tailings substrates will also be discussed.

\section{$2 \quad$ Evaluating shear effects in polymer treatment of substrates}

\subsection{Overview}

To provide a foundational exploration of the effects of shear on polymer-treated substrates, seven polymers with anionic charge density of approximately $30 \%$ and intrinsic viscosity (IV) ranging between 9 and $19 \mathrm{dL} \mathrm{g}^{-1}$ were used to treat two synthetic slurries of differing solids content. IV is related to a polymer's average molecular weight, $M_{w}$, by the equation $[\eta]=K M_{w}{ }^{\alpha}$, where $K$ and $\alpha$ are constants. Throughout the paper, these slurries are referred to as 'model' substrates as they were developed to represent two points in the range of low to high-solids content slurries that can be encountered in the processing and treatment of mineral wastes. The paper focuses on the key testing and associated parameters needed to evaluate flocculation response of the various polymers in these model systems. The findings from a preliminary evaluation of the shear performance of two polymers with a $30 \%$ charge density, but molecular weight in the IV 14 and IV 15 range used to treat two different oil sands substrates, will also be discussed.

\subsection{Examining applied shear in model slurry systems}

The traditional approach to evaluating flocculants applied in industrial thickeners is using cylinder tests. They are relatively simple to conduct and can be used to rapidly screen the effectiveness of several flocculants to treat given substrates. However, reproducibility of these measurements can be challenging, with the results depending on both experimental technique and on the operator. To impart greater reliability and consistency in the polymer screening process, a shear vessel (Figure 1(a)) was used to evaluate treatment of the low-percent solids slurry (LPSS, 6 wt\%), while a topological mixer (Figure 1(b)) was used with the high-percent solids slurry (HPSS, $21 \mathrm{wt} \%$ ). They enable detailed evaluation of several factors that significantly influence flocculation performance which cannot be studied effectively with traditional cylinder tests. These include the effect of agitation intensity, residence time, aggregate strength, and multi-stage flocculant addition.

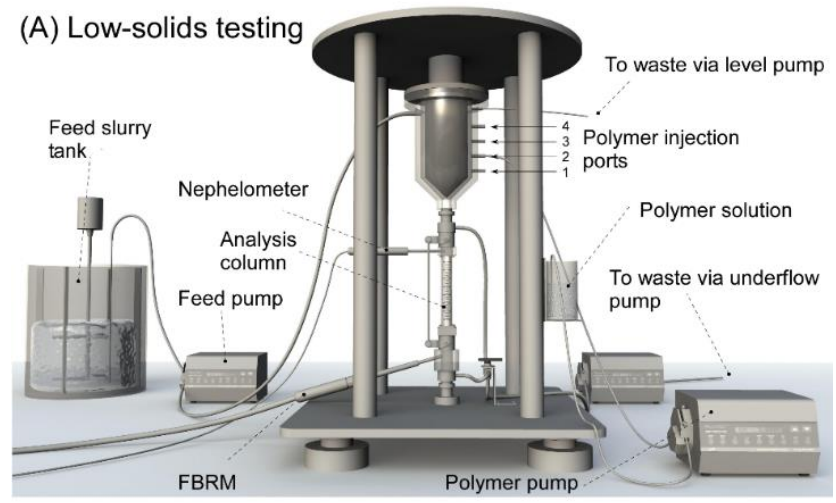

(B) High-solids testing

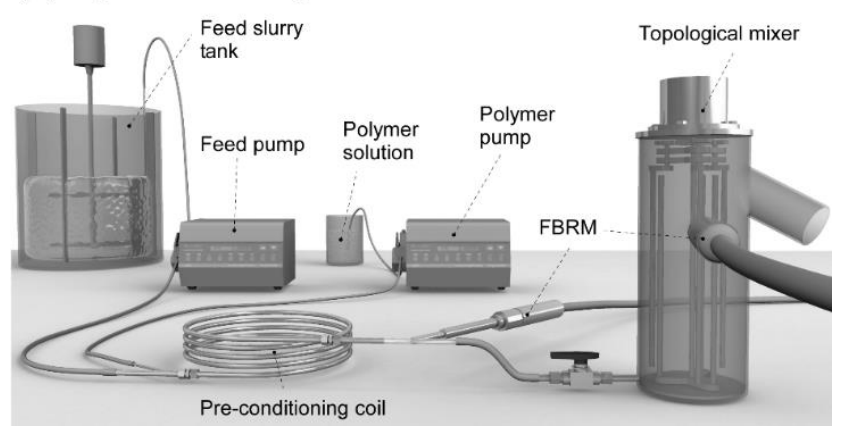

Figure 1 (a) CSIRO shear vessel for low-solids continuous flocculation; and, (b) Topological mixer for high-solids polymer addition 
The shear vessel consists of an outer fixed cylinder (ID $110 \mathrm{~mm}$ ) and an inner rotating cylinder (OD $100 \mathrm{~mm}$ ) driven by a variable speed motor. An annular gap of $5 \mathrm{~mm}$ exists between the cylinders. The inner cylinder is constructed from stainless steel and the outer cylinder of clear acrylic. The conical section at the base of the cylinders has a pitch of $45^{\circ}$ and maintains the $5 \mathrm{~mm}$ annular gap spacing. Flocculant inlets (ports one to four) are positioned on one side of the outer cylinder, allowing the reaction time under the applied agitation to be varied (port two was used in this study, giving a residence time of $16.5 \mathrm{sec}$ ). The slurry inlet port is positioned on the side opposite from the flocculant ports. LPSS was pumped from the feed tank to the shear vessel using a peristaltic pump at a constant delivery rate of $300 \mathrm{~mL} \mathrm{~min}^{-1}$. Dilute flocculant delivery was made via port two of the outer acrylic cylinder using a second pump. The agitation conditions experienced by the suspension in the annular gap are controlled by the rotation of the inner cylinder, the agitation intensity being directly proportional to the rotation speed. In the current study, the shear vessel was used at low, medium, and high mixing intensities of 150,250 , and $400 \mathrm{rpm}$, respectively.

The flocculated slurry flows through a glass analysis column, valved at both ends, which is fitted to the base of the cylinders. Settling rates can be measured in this column with continuous flow maintained by using a diversion line. A focused beam reflectance measurement (FBRM) probe was inserted below the analysis column at an angle of $70^{\circ}$ to the flow, allowing material to be suitably presented to the probe window but preventing any solids build-up on the probe. A second peristaltic pump was used to control the underflow flow rate and was set to draw $95 \%$ of the total incoming flows. This allowed for approximately $5 \%$ of the flow to report to a levelling outlet which maintained a constant volume of slurry within the vessel. Low (IV 10) and high (IV 18) molecular weight (MW) anionic polyacrylamide (PAM) polymers were assessed in this manner, using hindered settling rate as the performance measure. Flocculated slurry was sampled for floc density analysis at a point below the shear vessel analysis column and before the underflow pump (Figure 1(a)).

Challenges associated with bench-scale testing of polymer reagents (Fawell et al. 2015) are exacerbated in high-solids tailings applications. To this end, attention was paid to the mode of polymer addition and mixing with the HPSSs. High-solids slurries were treated with polymers in a two litre chaotic or topological mixer (Figure 1(b)). To conduct this experiment, kaolin slurry and polymer solution at the desired flow rate were pumped to separate inlets offset by $180^{\circ}$ at the base of a two litre acrylic mixing vessel. The conditioned slurry rises and swirls in the topological mixer due to the cycloid motion of the vertical rods while the high solution viscosity slows the aggregation rate. The thickened slurry exits the vessel via an angled overflow pipe ( $4 \mathrm{~cm}$ diameter). An adaptor oriented approximately $90^{\circ}$ to the slurry overflow pipe enabled a G400 FBRM probe $(0.95 \mathrm{~cm}$ diameter) to be inserted into the mixing medium.

The topological mixer achieves rapid mixing of HPSSs under low-shear conditions through the slow braiding motion of six vertical rods ( $28 \times 0.6 \mathrm{~cm}$ diameter) (Finn \& Thiffeault 2011). Three outer gears, each bearing two vertical rods, rotated around a larger inner gear, ensuring that the mixer provided a constant, reproducible shear history to the entire slurry (Costine et al. 2014). To examine the effect of tapered-shear mixing on dewatering performance, the slurry and polymer solution were first pumped through a spiralled coil $(7.7 \mathrm{~mm} \mathrm{ID)}$, with the pre-aggregated mixture then delivered to the base of the topological mixer (Figure 1(b)). Steady-state operation was confirmed when the FBRM statistics stabilised for the condition under study (i.e. constant mean aggregate sizes in the topological mixer). A sample of thickened slurry was then collected for a $24 \mathrm{hr}$ gravity drainage test using a $4^{\circ}$ slope (Costine et al. 2016). The percentage of total water recovered was calculated from a mass balance of the release water and the water remaining within the sediment.

\subsubsection{Polymer solution and slurry preparation}

BASF synthesised seven polyacrylamide-type polymers of a fixed anionic charge density ( $30 \%)$ with IV ranging between 9 and $19 \mathrm{dL} \mathrm{g}^{-1}$. This range represents what are typically referred to as low to high IV polymers. The appropriate mass of dry powder polymer was added to the vortex produced by an overhead stirrer rotating at $250 \mathrm{rpm}$ in the volume of deinonised water required to achieve the desired polymer concentration. Working polymer concentrations (0.4-1.0 wt\%) were determined to maintain a constant solution viscosity (and minimise dispersion effects), with the low MW polymers being applied at a higher concentration than the high MW 
polymers. Rapid stirring was continued for $30 \mathrm{~min}$, with stirring then reduced to a rate of $80 \mathrm{rpm}$ to provide continuous movement of the hydrated polymer solution. This gentle agitation was maintained for $16 \mathrm{hrs}$. The anionic copolymer solution was prepared fresh every three days to minimise aging effects. Polymer dosage is expressed as grams of polymer added per tonne of dry kaolin solids $\left(\mathrm{g} \mathrm{t}^{-1}\right)$.

The HPSSs ( $21.3 \mathrm{wt} \%$ solids) were made up in a $0.005 \mathrm{M} \mathrm{CaCl}_{2}$ solution and stirred at $250 \mathrm{rpm}$ for $8 \mathrm{hrs}$ and allowed to equilibrate while sitting for two days. The LPSSs ( 6 wt\% solids) were prepared in $0.035 \mathrm{M} \mathrm{NaCl}$ solution. It should be noted that substrate slurries are never totally reproducible from batch-to-batch and some degree of natural aggregation is observed in all clay-based mineral tailings. To avoid effects of variability between slurry batches, all direct comparisons of polymers or their blends were performed on a single slurry batch.

\subsubsection{Aggregate/s/urry characterisation}

\subsubsection{Focused beam reflectance measurement}

A Mettler Toledo model G400 FBRM with PI-14/206 probe was used to monitor aggregate sizes as described in Heath et al. (2002). FBRM utilises a monochromatic laser beam scanning a circular path and focused at the external surface of a sapphire window. Reflections from particles or aggregates are measured as pulses when they exceed a threshold intensity, and thousands are measured every second, generating an unweighted chord length distribution. A chord length is the straight-line distance from one edge of a particle or aggregate to another edge. Chord length distributions from 1 to $4,000 \mu \mathrm{m}$ were recorded every $2 \mathrm{sec}$ and averaged over five measurements when stability had been achieved for the condition being studied.

\subsubsection{Aggregate density analysis}

The size, shape, settling velocity, and density of individual aggregates present within the various treated slurries were determined using a floc density analyser (FDA) (Farrow \& Warren 1993). Each slurry sample was diluted with its filtered release water to a solids content of approximately $0.1 \mathrm{wt} \%$, taking care to minimise aggregate rupture. The temperature of the dilute suspension at the time it was added to the FDA cell was $24^{\circ} \mathrm{C}$. The valves of the analysis chamber were closed to completely isolate the suspension and yield a convection-free settling chamber. A camera with special lenses recorded aggregate sedimentation under the influence of gravity within the analysis cell. The size of individual aggregates was determined by assuming an overall ellipsoidal geometry defined by maximum horizontal and vertical dimensions (with respect to the direction of settling). The diameter of the sphere which had a Stokes settling velocity equivalent to the ellipsoid was determined using the expressions given by Happel and Brenner (1973). An effective aggregate density was calculated using a terminal velocity equation (with an estimated spherical diameter) based on Stokes' law, from knowledge of the size, settling velocity and the viscosity and density of the fluid. Aggregates with tails or filaments were not measured. Digital imaging and semi-automated image analysis software facilitated strong statistical comparisons of hundreds of individual aggregates.

\subsubsection{Particle vision and measurement (PVM)}

Images of individual aggregates present within different sediments were acquired with a Mettler Toledo particle vision and measurement (PVM) probe. Particle vision and measurement provides a focused strobing laser illumination of a stirred $0.5 \mathrm{wt} \%$ slurry capturing an area of $660 \times 880 \mu \mathrm{m}$ down to a $5 \mu \mathrm{m}$ resolution. A special lens and lighting configuration provided a limited depth of field, such that any aggregate in focus could be sized correctly. The PVM images were altered to enhance contrast and the colours inverted to imitate the back-lighting conditions used in the FDA.

\subsubsection{Compressibility and rheology measurements}

A stepped-pressure filtration rig (the Permacon 3000) was used to apply pressure to slurry contained in a compression cell, forcing liquid out through a filter membrane at the cylinder base (Usher et al. 2001). The pressure of filtration is monitored using a pressure transducer mounted flush in the piston face. The rate 
of filtration is monitored through the rate of movement of the piston and the movement of this piston is tracked using a linear encoder with a spatial resolution of $10 \mu \mathrm{m}$. The compressibility test is conducted as a normal batch filtration experiment at the lowest pressure desired until equilibrium is reached. This process is repeated at incrementally higher pressures until equilibrium is achieved. After each pressure step, the resulting cake at its characteristic solids concentration $\left(\phi_{\infty}\right)$ will have a compressive yield stress $\left(P_{y}(\phi)\right)$ equivalent to the applied pressure such that:

$$
\Delta P=P_{y}\left(\phi_{\infty}\right)
$$

Vane yield stresses after deposition were measured with a Haake VT550 rheometer using an FL100 vane controlled at $0.3 \mathrm{rpm}$ for $60 \mathrm{~s}$ using RheoWin Pro software (Thermo Haake $\mathrm{GmbH}$ 2017). Vane measurements are preferred for slurries treated with high polymer dosages, but do not provide any information on the response to shear rate. More information can be obtained using concentric cylinder (cup-and-rotor) sensors that allow shear stress to be measured as the rotation rate of the inner cylinder (i.e. the shear rate) is ramped up. Unfortunately, such measurements become problematic in the current application, given the likelihood of shear thinning and the sensitivity of aggregates to shear history.

\subsection{Evaluating shear effects in treating oil sands slurry}

\subsubsection{The quest for 'optimal mixing' and 'optimal dose'}

In addition to the challenges associated with bench-scale polymer tests noted earlier in this paper, evaluation of polymer treatments for oil sands tailings slurries presents additional challenges. These challenges are largely associated with the nature of oil sands substrates which often have one or more of the following: solids contents exceeding $20 \mathrm{wt} \%$; mineralogical clay content exceeding $40 \%$; residual emulsified bitumen; and in the case of aged fluid fine tailings (FFT), the rheological property of thixotropy, or a time-dependent response to applied shear. In this paper, the oil sands tailings slurry investigated would historically be described as mature fine tailings (MFT) and had the following characteristics: $33 \mathrm{wt} \%$ solids content ( $100 \%$ fines with clay content of $77.5 \%$ using methylene blue index, $3 \mathrm{wt} \%$ bitumen, and a specific gravity of 1.2. The polymers were both made down with process water.

Most lab-scale screening of polymer treatments for oil sands substrates involves shear thinning FFT to break down its intrinsic structure to achieve a constant viscosity that facilitates efficient mixing with the hydrated polymer solution being evaluated. Care must be paid to pre-shearing of the oil sands substrate, adequate hydration of the polymer solution, and applied mixing energy.

The process of screening polymer treatments typically involves placing a known mass of shear-thinned/ shear-conditioned FFT on a variable height platform that is centred on the flat blade impeller installed in a variable speed overhead mixer. Once the impeller has achieved the desired speed (typically upwards of $200 \mathrm{rpm}$ ), the dose of hydrated polymer is added to the vortex created by the rotating impeller. Using a rheometer, the peak stress can be identified, as it is typically tagged as the point immediately preceding where 'peak structure' resulting from flocculation occurs. The resulting treated slurry is then placed on a sieve and the amount of water initially released and at $24 \mathrm{hrs}$ is measured. Internal testwork focused on PAM treatment of similar FFTs suggests that free-draining water released within the initial $24 \mathrm{hr}$ period post polymer treatment generally exceeds $95 \%$, with the remaining free draining water taking up to two weeks to be available for collection (Boxill 2016). Thus, for practical purposes, polymer treatment producing the highest solids content and corresponding highest net water release at $24 \mathrm{hrs}$ is typically selected as the candidate chemistry to be advanced for evaluation at the field-scale.

Consequently, the primary challenge with the current method of chemistry selection to treat oil sands substrates is that replicating the substrate conditioning and polymer mixing used in the lab is significantly more challenging in a field context, especially when substrate variability is the norm and a range of operational conditions must be managed. Therefore, in the hopes of increasing the likelihood that a selected polymer will perform as desired in the field, increasing attention is being paid to ensure that recommended 
polymers can effectively treat these substrates over a much wider window of substrate characteristics and operating conditions.

\subsubsection{Preliminary investigations completed with treated oil sands slurry}

A preliminary investigation into the effects of mixing on polymer-treated oil sands substrates was completed using torque data to characterise the shear resistance profile of two copolymers and blends of them at various ratios. An IKA Eurostar 60 set at a mixing speed of $320 \mathrm{rpm}$ was used to mix the copolymers into the pre-sheared substrate and to collect torque data. The torque profile was recorded during each test and the mixer's data logging software was used to monitor the increase in torque during structure development, peak structure, and as the polymer-treated substrate became shear thinned.

The effect of post-flocculation shear on the ability of polymer-treated oil sands substrates to continue effective dewatering was also investigated. In this experiment, the settling performance of an oil sands substrate that received a single copolymer treatment and subsequently sheared was compared to the settling performance of samples of shear-treated substrate that received a range of secondary doses of copolymer.

\section{$3 \quad$ Results}

\subsection{Effect of applied shear during low-solids substrate flocculation}

To contrast conventional polymer-bridging flocculation at typical feedwell solids concentrations with inline polymer addition to high-solids substrates, the continuous Couette flocculation of a tailings analogue at 6 wt\% solids was examined using the shear vessel technique described earlier.

The full dosage response curves (Figure 2) for low and high MW PAMs across different shear regimes are provided, using hindered settling rate as the performance measure. The results cover dosages from the onset of aggregation through to achievement of settling rates $\left(>30 \mathrm{~m} \mathrm{hr}^{-1}\right)$ which are well above what are usually required in practice. In terms of the dosage requirement to give a target settling rate, it was found that the low MW PAM out-performed the high MW PAM under mild mixing (150 rpm), with little difference in the dosage needed to produce a settling rate of approximately $20 \mathrm{~m} \mathrm{hr}^{-1}$. At $250 \mathrm{rpm}$, the dosage required by the low MW PAM to give a settling rate of $20 \mathrm{~m} \mathrm{hr}^{-1}$ was $30 \%$ more than the dose of high MW PAM needed to produce the same effect. Increasing the applied shear to $400 \mathrm{rpm}$ created a $42 \%$ difference between the dosages of low MW and high MW polymer.

When more intense shear was applied, the shorter chains of the lower MWs did not readily support creation of larger aggregates able to survive high-shear flocculation conditions, as indicated by real-time FBRM monitoring of the aggregation state (Figure 2(b)). Whilst the unweighted distributions (inset) are more sensitive to the presence of residual fines, applying a length weighting to the data produces distributions that are more in keeping with the volume-based distributions obtained from laser light scattering techniques. Under low fluid shear (150 rpm), the mean square-weighted chord lengths recorded for IV 10 and IV 18 were similar ( $805 \mathrm{cf} .942 \mu \mathrm{m}$, respectively), whereas under intense mixing $(400 \mathrm{rpm})$, the much larger sizes created by the longer chain ( $593 \mathrm{cf} .1,058 \mu \mathrm{m}$ ) gave access to faster settling rates. 

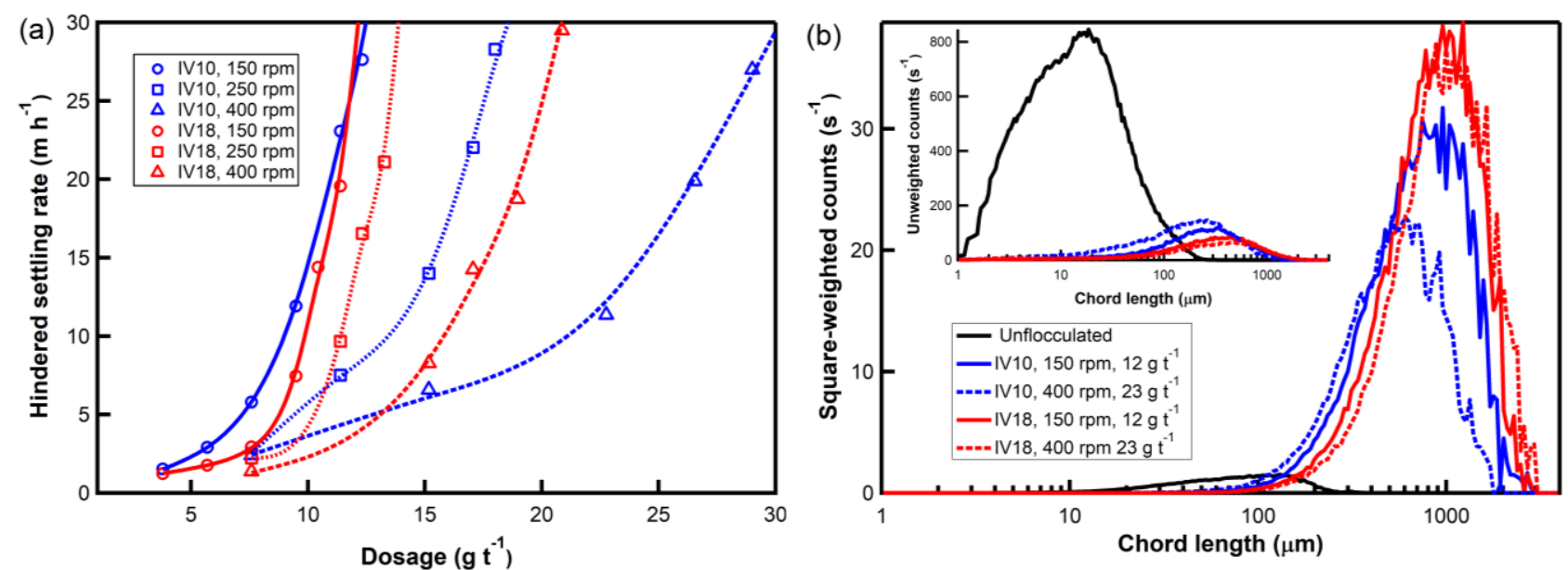

Figure 2 (a) Effect of increasing mixing intensity on the hindered settling rate of $6 \mathrm{wt} \%$ tailings surrogate for low (IV 10) and high (IV 18) MW PAM as a function of applied dosage; and, (b) The corresponding FBRM chord length distributions

It should be noted that because flocculant dosage is achieved using a specified mass of flocculant, the lower MW PAM had about twice as many polymer chains available compared to an equivalent mass of high MW PAM, with the effects of this best seen during the initial stages of flocculation. It is also noteworthy that the LPSS tailings surrogate appears to be easier to flocculate than slurry prepared at an equivalent total solids concentration with kaolin alone (Costine et al. 2017). While aggregation response is dominated by the clay fraction, the presence of a coarse quartz phase reduces required dosages and leads to high settling rates.

A study examining the continuous Couette flocculation of kaolin slurry using seven anionic PAMs with different MWs but the same chemistry allowed the flocculant dosage response curves to be delineated for nine distinct mixing conditions by varying agitation intensity and duration (Costine et al. 2017). For a given solids concentration, the potential for bridging and the strength of adsorption varies with increasing MW. Consequently, the distance between the mineral particles within an aggregate and the packing density of particles incorporated into an aggregate are likely to change for flocculants with the same chemistry but different MW. The hindered settling rates shown in Figure 2 reflect changes in both the aggregate size and structure (density). The effect of flocculant MW and mixing conditions on aggregate densities was explored in greater depth using the FDA technique.

Samples of flocculated slurry were collected at a point below the shear vessel analysis column and before the underflow pump (Figure 1(a)) for each of the IV 10 and IV 18 PAMs at 150, 250, and $400 \mathrm{rpm}$. PAM dosage was adjusted to give average free-settling rates less than $7 \mathrm{~m} \mathrm{hr}^{-1}$ across the range of agitation intensities studied. Aggregate sizes in each test ranged between 50 and $400 \mu \mathrm{m}$. The polydisperse nature of the primary particles in the suspension resulted in data scatter which required use of best fit curves to interpret results (not presented due to limited space). Under mild mixing $(150 \mathrm{rpm})$, the greater number of bridging connections produced by the low MW flocculant resulted in more compact, slightly higher effective aggregate density which produced faster settling aggregates than equivalent aggregates formed by the high MW flocculant under the same mild mixing condition. Additionally, examination of the free-settling behaviour of flocculated structures produced under different shear regimes confirmed that structures formed by low MW PAMs under mild mixing have less intra-aggregate liquor than those formed by high MW PAMs.

However, the effect of increasing mixing intensity on aggregate densification was more pronounced for the higher MW at mixing speeds of $250 \mathrm{rpm}$ and greater. This finding suggests that re-arrangement of the partially adsorbed flocculant occurred more slowly for the longer chain high MW PAMs when used to treat the synthetic LPSS tailings substrate. 


\subsection{Effect of applied shear during high-solids substrate flocculation}

The two main methods for inline mixing of polymer solution with fine particle tailings are dynamic mixing and static inline mixing. Dynamic mixing utilises a motor-driven mixing device such as an impeller to induce fluid mixing, while static inline mixing uses the energy generated in the pipe as it conveys the fluid to mix the polymer and tailings. Given that mixing energy is directly coupled with flow rate for a static mixing system, and mixing efficiency is impacted by fluid dynamics, improper design can lead to unstable and suboptimal mixing. Mixing needs to be consistent across a wide range of processing conditions, including fluid rheology and flow rate. For inline mixing, attention needs to be paid to the balance between adequate polymer-slurry mixing and the shear sensitivity of the aggregate structures formed. The completed study aimed to quantify the evolution of aggregate sizes, rheological strength, and compressibility with increasing residence time under low shear in the topological mixer.

During the test, flow rates were varied between 0.15 and $1.3 \mathrm{~L} \mathrm{~min}^{-1}$ which allowed the residence time of the HPSS in the topological mixer to range between 1.6 and $13.8 \mathrm{~min}$. The main characteristics of the dewatering performance were directly correlated to the FBRM mean chord lengths, with maximum water returns and aggregate sizes recorded after about 3-4 min. Longer residence time reduced the apparent aggregate size, diminished water return, and slightly increased the overall final solids concentration. This finding highlights the importance of applying the correct shear regime during inline polymer addition. Whilst some degree of aggregate re-arrangement and breakdown is important for maximising water release, extending the mixing too long leads to over-shear, with little additional water release (Wells et al. 2011).

Photographs of selected thickened high-solids slurries after deposition on a screen with $1 \mathrm{~mm}$ opening are shown in Figure 3 to illustrate some of the general trends observed with respect to increasing residence time under low-shear mixing conditions. PVM images provide additional insight into the overall morphology of the aggregates formed at each mixing time. Residence times under $1.9 \mathrm{~min}$ did not produce the desired continuous network structure with many fines passing through the $1 \mathrm{~mm}$ screen. As seen in the associated PVM image, these small, fractal aggregates showed numerous filaments or tails. This contrasts the larger, and more compact aggregate structures produced between 3 and $7 \mathrm{~min}$. Additional mixing time up to $13.8 \mathrm{~min}$ released some fines but an overall dense, rigid structure was maintained.

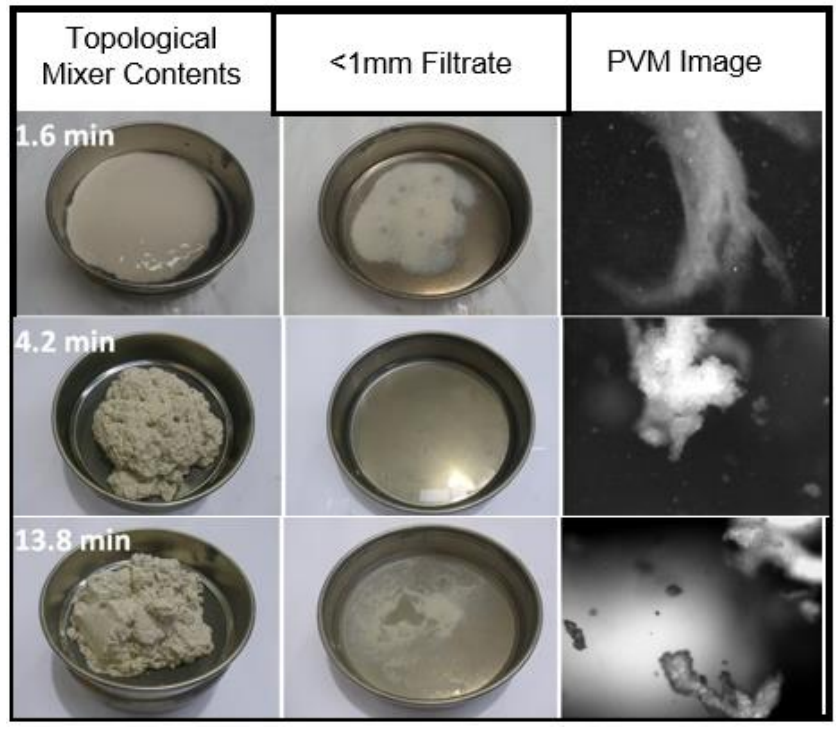

Figure 3 Photographs and PVM images as a function of residence time after treatment of $21.3 \mathrm{wt} \%$ kaolin with a medium (IV 14) MW PAM at $1,000 \mathrm{~g} \mathrm{t}^{-1}$

Using the FBRM to measure the chord length of aggregates throughout mixing of the kaolin slurry treated with a medium MW polymer at $1,000 \mathrm{~g} \mathrm{t}^{-1}$, it was observed that while irreversible aggregate rupture was observed at mixing times exceeding $4 \mathrm{~min}$, the degree of rupture of the aggregates formed was not excessive. 
Consequently, the aggregates formed under low-shear conditions exhibit moderate strength with ruptured structures increasing the number of aggregates between 50 and $500 \mu \mathrm{m}$.

Figure 4 shows the effect of residence time on the compressive yield stress-volume fraction relationship for the same treated kaolin slurry. Although it is common for industry to use weight percent solids to describe the solids fraction in a particulate suspension, volume fraction is used because it is this parameter used in most dewatering models (Hogg et al. 2005).

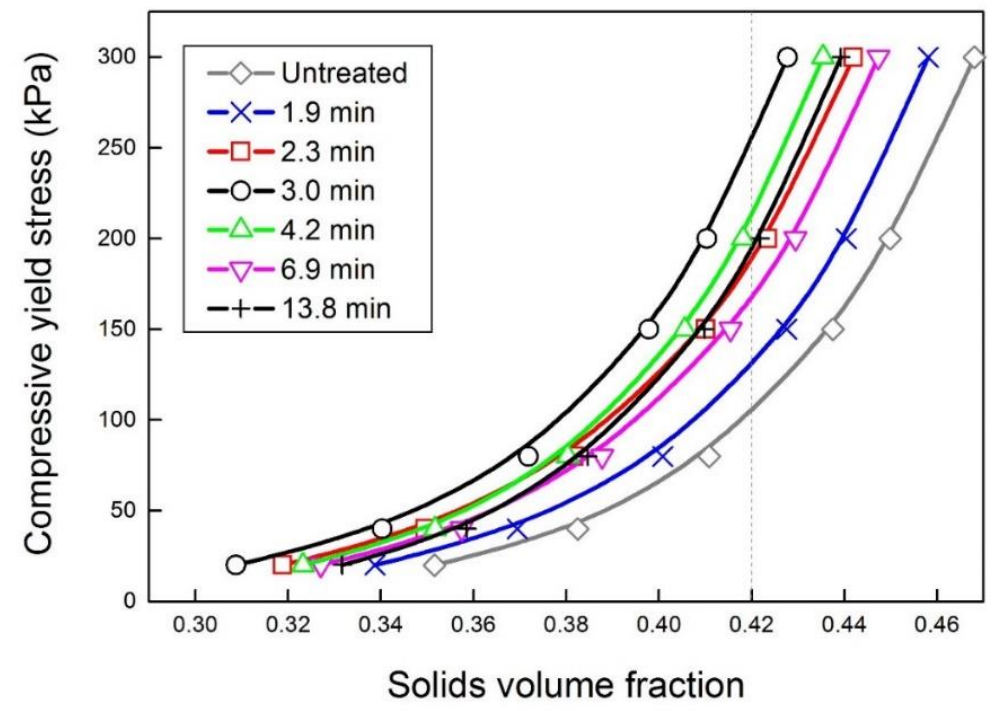

Figure 4 Compressive yield stress curves from stepped-pressure experiments as a function of residence time for $21 \mathrm{wt} \%$ kaolin treated with a medium (IV 14) MW PAM

A plot of compressive yield stress versus solids volume fraction indicated that as conditioning time increases, the curves shift left to a higher compressive yield stress relative to untreated kaolin, with the greatest shift occurring at $3 \mathrm{~min}$. Longer conditioning times (greater than $3 \mathrm{~min}$ ) reduced the strength of the network as well as the amount of compression required to effect volume change. For example, the compressive yield stress halved after 6.9 min of low-shear conditioning, with only minimal effect on water recovery and final solids concentration (Figure 4).

\subsubsection{Tapered-shear mixing}

Tapered-shear mixing has been tested in a range of applications, from the coagulation of dilute kaolin slurries with alum (Francois et al. 1981) to large-scale operations in water treatment using baffled hydraulic channels (McConnachie \& Liu 2000). However, little information is available on applications relevant to the mineral processing industries. A tapered-shear mixing regime was tested where an initial rapid dispersion of the polymer chains within the slurry gave way to a subsequent low-shear conditioning environment. Initial mixing of the kaolin HPSS with a medium MW polymer dosed at $1,000 \mathrm{~g} \mathrm{t}^{-1}$ was provided by a series of $7.7 \mathrm{~mm} \mathrm{ID}$ stainless steel coils that could be connected to give any combined length ranging from 0.5 to $22 \mathrm{~m}$ (a $2 \mathrm{~m}$ coil was used for the experiment described in this paper). The HPSS was pumped at a rate of $1.1 \mathrm{~L} \mathrm{~min} \mathrm{~m}^{-1}$ through the $2 \mathrm{~m}$ length of coil producing $5 \mathrm{sec}$ of pre-aggregation time. Contents from the high-shear preconditioning coil were then pumped to the topological mixer where they experienced further conditioning under low shear.

Distinctly robust aggregate structures that rapidly expelled water were produced and allowed to overflow from the topological mixer's low-shear environment. Water recoveries after 24 hrs of gravity drainage with and without this tapered-shear mixing regime were 74 and $30 \%$, respectively, and the corresponding final solids concentrations were 44.3 and $30.8 \mathrm{wt} \%$. Treated aggregates produced using the tapered-shear method were also placed on screens with $1 \mathrm{~mm}$ openings and after $1 \mathrm{hr}$, the vane yield stress was measured as a function of the final solids concentration (Figure 5). 


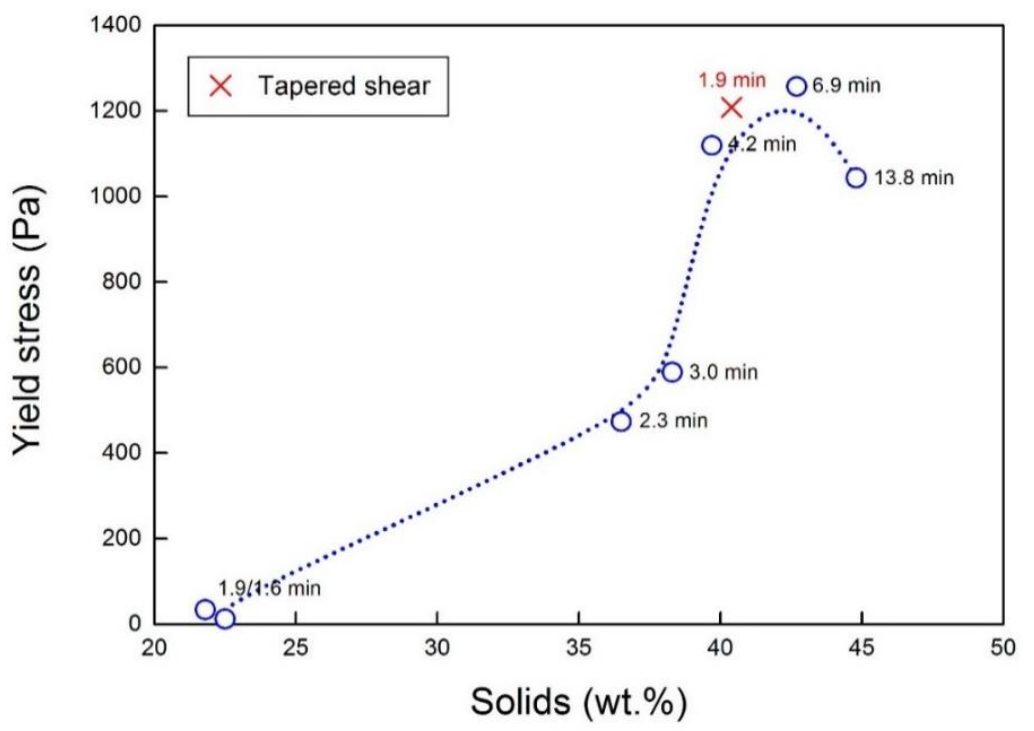

Figure 5 Effect of residence time under low-shear mixing conditions on vane yield stress measurements

The data points in Figure 5 are annotated with the conditioning times applied in the topological mixer. The data shows that applying a tapered-shear mixing regime produced a similarly dense, networked structure, offering the benefit of significantly reduced conditioning times under low shear.

\subsection{Shear effects in polymer treatment of oil sands tailings}

\subsubsection{Effects of polymer type and combination on treated substrate torque data}

Evaluation of optimal mixing energy and optimal dose of polymer used to treat oil sands tailings tends to focus on maximising water release under assumed constant mixing conditions for a fixed treatment system, either at a specified single 'optimised' dosage or for a narrowly defined range of polymer dosages that are assumed to vary primarily depending on the clay content of the input tailings substrate. However, given the variability that often exists for a range of parameters within the oil sands tailings treatment context, differences between lab-based results and measured field performance data can be significant. The investigation completed was entirely lab-based and sought to identify whether two anionic polymers of similar intrinsic viscosity but each having a different primary ion, would exhibit differences in torque behaviour individually and when used in combination to treat a selected oil sands tailings slurry.

Comparison of the torque profiles of the unblended polymers reveals that even in this simplistic evaluation, each chemistry exhibits differences in its responses to shear applied at $320 \mathrm{rpm}$ with a flat blade impeller driven by an overhead mixer. Standard PAM polymer produces a single peak torque response over the range of dosages tested with peak torque ranging from approximately 13 to $23 \mathrm{~N} \mathrm{~cm}^{-1}$ and occurring between 8 and $25 \mathrm{sec}$. For this chemistry, both the time to peak torque and the magnitude of peak torque increased as the polymer dosage increased from $965 \mathrm{~g} \mathrm{t}^{-1}$ (\% wt solids basis) to $1,448 \mathrm{~g} \mathrm{t}^{-1}$. In contrast, FFT treatment with a novel polymer produces differences in the shape of the post-treatment torque profile based on dose. At polymer doses between 1,048 and $1,235 \mathrm{~g} \mathrm{t}^{-1}$, the novel polymer exhibits a single peak torque that ranges from 14 to $17 \mathrm{~N} \mathrm{~cm}^{-1}$ occurring between 8 and $12 \mathrm{sec}$. However, for polymer dosages ranging between 1,347 and $1,544 \mathrm{~g} \mathrm{t}^{-1}$, the novel polymer exhibited both an initial peak followed by a larger secondary peak torque often occurring at several multiples of the time when the lower initial peak torque was observed (Figure $6(b))$.

For the novel polymer at the higher dosages indicated, the initial peak torque ranged between 7 and $10 \mathrm{~N} \mathrm{~cm}^{-1}$ and occurred between 5 and $15 \mathrm{sec}$ after the start of the test. The secondary peaks at these dosages ranged between 14 and $18 \mathrm{~N} \mathrm{~cm}^{-1}$ and occurred between 45 and $115 \mathrm{sec}$. While the peak torque exhibited by both polymers is technically equivalent, the longer duration of mixing time until the peak torque recovers 
suggests that the novel polymer chemistry may tolerate either extended mixing or applied shear and still produce the same level of dewatering achieved by the standard polymer. In other words, the novel polymer appears to exhibit some capacity to recover after it has been subjected to an extended period of shear, which could be beneficial in applications where treated FFT travels for extended distances in pipes prior to deposition in the tailings storage area (TSA).

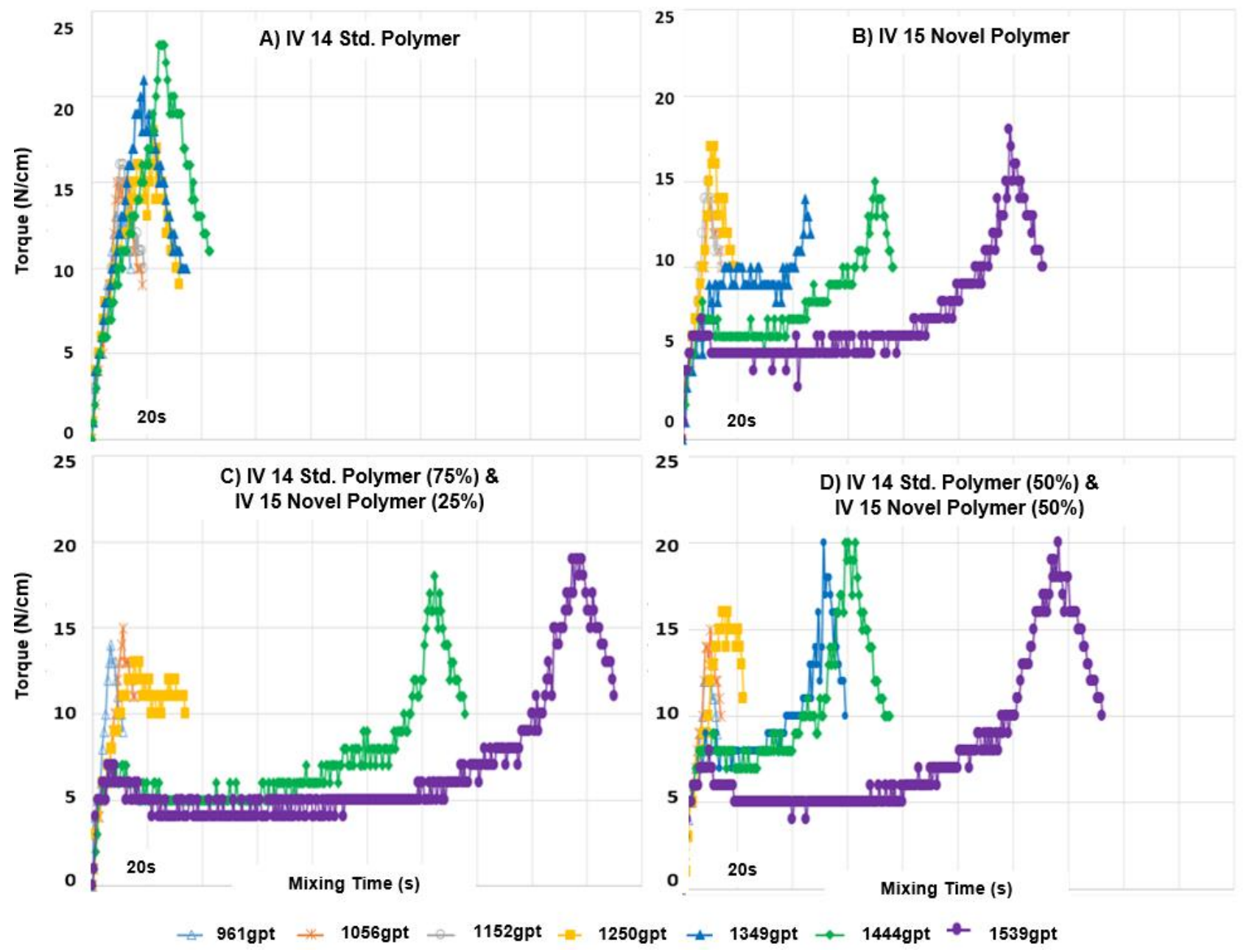

Figure 6 Torque profiles for two polymers with similar IV and mixed at different ratios (average concentrations for each dosage range shown)

It is interesting to note that substituting $25 \%$ of the standard polymer with the novel polymer produces the same secondary peak effect, but with a higher secondary peak than was achieved by the novel polymer on its own at combined polymer dosages between 1,349 and $1,539 \mathrm{~g} \mathrm{t}^{-1}$. It is also notable that the timing to the secondary peak in this copolymer mixture ranged from $52 \mathrm{sec}$ to approximately $137 \mathrm{sec}$. As the proportion of the novel polymer added increases to $50 \%$, the magnitude of the secondary peak becomes more consistent with the peak torque exhibited when only the novel polymer is used to treat the oil sands tailings substrate. However, it is notable that the timing of the secondary peaks in the 50:50 copolymer mixture ranged between 125 and $175 \mathrm{sec}$.

\subsubsection{Effects of post flocculation shear on substrate dewatering using different polymers}

The objective of the second experiment was to both replicate a real-world challenge faced by some operators and to investigate possible solutions. The challenge faced by some operators relates to inadequate accounting for the total mixing that a polymer-treated oil sands substrate will be subjected to. This often results in degradation and breakage of the initially flocculated material prior to deposition. Commonly, operators then evaluate candidates for secondary polymer addition just prior to discharge into the TSA using 
inline thickening techniques. The initial objective of this testwork was to first mimic the type of aggregate structure typically observed due to over-mixing of the initially treated FFT and then to identify an appropriate secondary dosage of polymer that would be needed to re-aggregate the degraded aggregates prior to discharge in the TSA. An oil sands substrate was treated with both the standard polymer and the novel polymer described in Section 3.2.1. To replicate the post-treatment degradation that was observed in the field, each of the treated samples were sheared using an overhead mixer until a minimum yield stress was achieved (i.e. total breakdown of the post-treatment aggregate structure) and then blended at a ratio of $50 \%$ by volume with process water. The total breakdown of the aggregate structure was intended to replicate the sort of breakdown that was observed in the field at the spigot discharge location into the TSA.

Portions of these samples were used to create the sample controls indicated in the lower portion of Figure 7. Subsamples of the novel polymer control were then treated with three different dosages of novel polymer to investigate the effect of secondary polymer addition to facilitate fines re-aggregation.

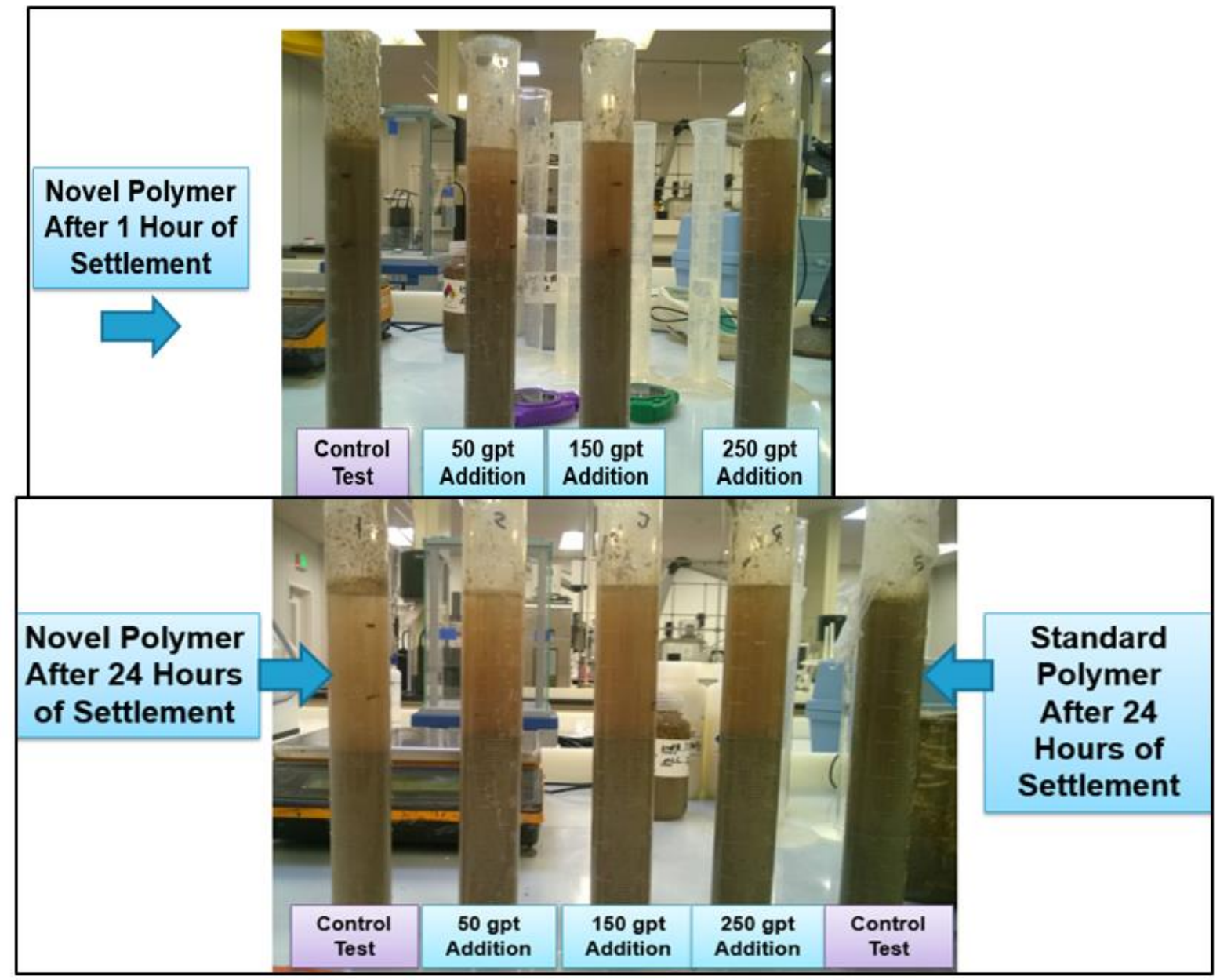

Figure 7 Comparison of settlement responses to various polymer treatments

As seen in Figure 7, after $1 \mathrm{hr}$, it appears that secondary addition of the novel polymer at dosages ranging between 50 and $150 \mathrm{~g} \mathrm{t}^{-1}$ produced favourable re-aggregation of the degraded treated tailings. However, after $24 \mathrm{hrs}$, while the clarity of the samples that received secondary polymer treatment improved, the reaggregation and clarity observed in the novel polymer control seemed to compare favourably with the samples that had received a secondary dosage of the novel polymer. 


\section{$4 \quad$ Closing discussion}

The mixing studies completed with kaolin slurries of varying total solids content highlight the importance of the mixing regime and conditions under which initial particle aggregation and aggregate growth occur. In both the shear vessel (used to evaluate the low-solids content slurries) and in the looped coil shearing (used to feed the topological mixer in some work with the higher solids content slurries), the annulus where both the polymer and untreated slurry are first introduced is highly controlled and notably small relative to the size of the ultimate chamber where aggregate conditioning occurs. In the shear vessel studies, it is notable that lower MW polymers appear to produce aggregates that achieve the highest settling rate under mild mixing conditions (around $150 \mathrm{rpm}$ ), while the higher MW polymers yield higher settling rates when higher mixing energies are used at a lower dosage than what can be achieved by a low MW under similar high-shear conditions.

Use of looped coil initial shearing followed by aggregate conditioning in the low-shear environment provided by the topological mixer suggests that water recovery and solids content from this tapered-shear configuration can be significantly improved when compared to systems that do not provide for aggregate conditioning under low shear. These findings suggest that having the correct residence time in a topological mixer, as well as optimised mixing of slurry and polymer, are critical to the performance of inline polymer injection. This is an important finding, since many inline flocculation applications do not feature a low-shear conditioning environment after the polymer has been injected into the pipeline. It is also notable how the use of tapered shear could significantly reduce the conditioning time needed to create large aggregates. This potentially provides greater flexibility for end-of-pipe dewatering schemes.

While the initial experiments completed with the oil sands slurries suggests that ionic compositional differences between the polymers effect both the magnitude of peak torque exhibited and the shapes of the torque profiles, insight from the work with the model slurry systems suggests that future experimentation examines a wider range of mixing environments to identify where the greatest aggregation occurs for treated oil sands slurries and the broader range of factors impacting aggregation. Even so, accounting for the shear environment that treated aggregates will be subjected to must also be included in the evaluation of suitable treatments, as the ultimate dewatering and aggregation of the deposited material is critical to the overall evaluation of polymer performance.

\section{References}

Beier, N, Wilson, W, Dunmola, A, \& Sego, D 2013, 'Impact of flocculation-based dewatering on the shear strength of oil sands fine tailings', Canadian Geotechnical Journal, vol. 50, no. 9, pp. 1001-1007.

Boxill, L 2016, The Impact of Fabric and Surface Characteristics on the Engineering Behaviour of Polymer-Amended Mature Fine Tailings, PhD dissertation, University of British Columbia, Vancouver.

Costine, A, Lester, D, Fawell, P \& Chryss, A 2014, 'Shear isn't mixing: how to build larger aggregates using chaotic advection for accelerated dewatering', in RJ Jewell, AB Fourie, PS Wells and D van Zyl (eds), Proceedings of the 17th International Seminar on Paste and Thickened Tailings, InfoMine Inc., Vancouver, p. 644.

Costine, A, Cox, J, Travaglini, S, Lubansky, A, Fawell, P \& Misslitz, H 2016, 'Influence of polymer molecular weight in low and high solids tailings applications', in S Barrera and RJ Jewell, (eds), Proceedings of the 19th International Seminar on Paste and Thickened Tailings, Gecamin, Santiago, chapter 4, paper 2.

Costine, A, Cox, J, Travaglini, S, Lubansky, A, Fawell, P \& Misslitz, H 2017, 'Variations in the molecular weight response of anionic polyacrylamides under different flocculation conditions', Chemical Engineering Science, vol. 176, pp. 127-138.

Farrow, JB \& Warren, LJ 1993, 'Measurement of the size of aggregates in suspension', in B Dobias (ed.), Coagulation and Flocculation - Theory and Application, Marcel Dekker, New York, pp. 391-426.

Fawell, PD, Costine, AD \& Grabsch, AF 2015, 'Why small-scale testing of reagents goes wrong', in RJ Jewell and AB Fourie (eds), Proceedings of the 18th International Seminar on Paste and Thickened Tailings, Australian Centre for Geomechanics, Perth, pp. 153-165.

Finn, MD \& Thiffeault, JL 2011, 'Topological optimization of rod-stirring devices', SIAM Review, vol. 53, no. 4, pp. 723-743.

Francois, R, van Haute, A \& Winderickx, G 1981, 'The influence of kinetic process parameters in flocculation and floc characteristics', Practical Theory of Flocculation Process: Proceedings of a Joint Seminar, pp. 103-118.

Happel, J \& Brenner, H 1973, Low Reynolds Number Hydrodynamics, Noordhoff International Publishing, Leyden.

Heath, AR, Fawell, PD, Bahri, PA \& Swift, JD 2002, 'Estimating average particle size by focused beam reflectance measurement (FBRM)', Particle \& Particle Systems Characterization, vol. 19, no. 2, pp. 84-95. 
Hogg, R, Lutsky, M \& Suharyono, H 1995, 'A simulation model for thickening of flocculated, fine-particle suspensions', in JA Herbst (ed.), Proceedings of the XIX International Mineral Processing Congress - Physical \& Chemical Processing, vol. 2, Society for Mining, Metallurgy \& Exploration, Englewood, pp. 81-86.

McConnachie, GL \& Liu, J 2000, 'Design of baffled hydraulic channels for turbulence-induced flocculation', Water Research, vol. 34, pp. 1886-1896.

McFarlane, A, Mensah, J \& Bremmell, K 2006, 'Optimizing flocculation and dewatering of clay dispersions via flocculant chemistry, temperature and pulp shear', Proceedings of the 23rd International Mineral Processing Congress, pp. 1,655-1,660.

Thermo Haake GmbH 2017, RheoWin Pro, Thermo Haake GmbH, Karlsruhe.

Usher, SP, De Kretser, RG \& Scales, PJ 2001, 'Validation of a new filtration technique for dewaterability characterization', AICHE Journal, vol. 49, no. 7, pp. 1561-1570.

Wells, PS, Revington, A \& Omotoso, $O$ 2011, 'Mature fine tailings drying - technology update', in RJ Jewell and AB Fourie (eds), Proceedings of the 14th International Seminar on Paste and Thickened Tailings, Australian Centre for Geomechanics, Perth, pp. 155-166. 